\title{
Modelagem de Processos Aplicada na Gestão de um Ambiente Real de TI
}

\author{
Rafael G. Ferreira ${ }^{1}$, Célia G. Ralha ${ }^{1}$ \\ ${ }^{1}$ Universidade de Brasília \\ Departamento de Ciência da Computação \\ Caixa Postal 4466 - 70.919-970 \\ Brasília - Brasil \\ gostinski@bb.com.br, ghedini@cic.unb.br
}

\begin{abstract}
This paper describes a study and a practical application of international Information Technology models at a real technology environment of one of the biggest banking institutions of the country. As an instrument of integration between the models and the real environment, we used the process modeling approach to help the management of information allowing the formalization of knowledge.
\end{abstract}

Resumo. Este artigo apresenta um estudo e uma aplicação prática de modelos internacionais de Tecnologia da Informação em um ambiente de tecnologia de uma das maiores instituições bancárias do país. Como instrumento de integração entre os modelos estudados e o ambiente real de TI, foi utilizada a abordagem de modelagem de processos, a qual auxilia de forma abrangente à gerência da informação além de possibilitar a formalização do conhecimento.

\section{Introdução}

Nas últimas duas décadas presenciamos grandes evoluções nas áreas de conhecimento relacionadas à gerência de Tecnologia da Informação (TI) e do conhecimento. Três fatores são essenciais para mudanças na forma de planejar, usar e extrair benefícios da TI: (i) a evolução dos modelos de gestão de TI aceitos internacionalmente; (ii) a evolução tecnológica que permite a integração destes modelos em ambientes organizacionais reais e (iii) o uso de indicadores e conceitos de governança nas práticas de gestão de TI.

Permitindo ligar todos essas evoluções nas áreas de conhecimento e estruturálas de forma prática, ágil e de fácil transmissão à empresa, os conceitos de processos, bem como as técnicas de modelagem de processos ocupam um lugar essencial. Inserido neste cenário, este trabalho aplica modelos internacionais como a biblioteca de melhores práticas de gestão de TI denominada Information Technology Infrastructure Library (ITIL) e o modelo de governança de TI denominado Control Objectives for Information and Related Technologies (COBIT). Foi utilizada também a abordagem de modelagem de processos no contexto organizacional de uma das maiores instituições bancárias do país.

O restante deste artigo está organizado da seguinte forma. A seção 2 traz uma breve revisão dos modelos baseados em processos que este trabalho aborda. Na seção 3 apresentamos a metodologia empregada para integrar os modelos abordados na seção anterior. A seção 4 descreve um estudo de caso realizado num ambiente real de TI de 
uma grande instituição bancária do país. Algumas conclusões e indicações de trabalhos futuros são apresentados na seção 5 .

\section{Processos e Modelos de TI}

As recentes discussões sobre processos possibilitaram inúmeras definições para o termo. Desde a definição de processo de negócio de [8], o qual cita que se trata de um conjunto de atividades estruturadas definidas para produzir uma saída específica; até outras variações como $[7,12]$ que definem processos de negócios utilizando um nível mais abstrato. Em [14] encontramos a definição de processo de negócio genérico baseada na cadeia de valor de Porter [15]. No escopo deste artigo nos referimos a processo como um conjunto de atividades que representam métodos de execução de um trabalho necessário para alcançar um objetivo organizacional. Esta definição é muito semelhante às definições apresentadas em $[11,6,13]$ e se torna interessante na medida que transcende áreas específicas, consome recursos e usa informações como meio de gestão de TI. Qualquer procedimento organizacional está sempre sustentado por um ou mais processos.

Neste cenário de revolução de processos organizacionais surge em 1990 o conceito de reengenharia do trabalho. Segundo [10], a idéia de reengenharia de trabalho era "não automatize, elimine". Ou seja, o autor que primeiro citou o tema de reengenharia do trabalho foca a necessidade de uma análise dos processos organizacionais ao invés de uma simples automação de procedimentos. Surgiu então o conceito de reengenharia de processos organizacionais ou Business Process Reengineering (BPR). Segundo [9], BPR significa uma filosofia de melhoria de performance empresarial através do redesenho ou reestudo dos processos envolvidos na organização.

No escopo deste artigo, utilizamos o conceito de modelagem de processos composto por etapas desde o levantamento das características de procedimentos até a interação de tarefas realizadas nos mais diversos níveis organizacionais, que através de uma modelagem possam ser alvo de análise e estudo. Tais etapas dos procedimentos relacionados à modelagem de processos proporcionaram o respaldo e a base necessários para elaboração e implantação de novos modelos processuais mais otimizados e eficientes.

Segundo [9] as fases genéricas de modelagem de processos são: (i) criação da visão empresarial; (ii) identificação e compreensão dos processos existentes; (iii) redesenho de processos; (iv) implementação dos processos e (v) manutenção dos processos. Neste contexto, o uso de um software para modelagem de processos facilita a formalização dos processos para uma melhor análise dos mesmos. A fase (ii) se dá através da elaboração de um modelo denominado as-is. Nesse modelo, procura-se atingir o máximo de fidelidade com que as tarefas são desempenhadas pelos atores; uma vez que esse modelo representa uma formalização do fluxo do processo através de uma documentação. Após a elaboração do modelo as-is pode-se iniciar as atividades de análise pela equipe envolvida no projeto de modelagem de processos.

Durante essa análise serão levantados os caminhos críticos, os gargalos, os desperdícios de recursos, as ambigüidades existentes, bem como a inexistência destas. Essas informações servirão de subsídio e respaldo formal na adoção de novas técnicas e metodologias visando uma otimização do processo em questão, através da adoção de técnicas de simulação. Com base nessa análise, constrói-se um novo modelo denominado to-be. $\mathrm{O}$ modelo to-be traz representado as mudanças que serão implantadas no modelo de gestão 
futuro. O método de modelagem de processos apresentado possibilita a integração entre modelos de gestão e governança de TI, os quais passaremos a expor através de uma breve visão por motivos de restrição de espaço.

\subsection{Modelo ITIL}

A biblioteca ITIL de infra-estrutura de TI é composta por um conjunto consistente de melhores práticas mundiais. Seu objetivo é alinhar os serviços de TI aos requisitos de negócios através da gestão de qualidade de seus componentes e serviços. O Modelo ITIL é dividido basicamente em dois grandes grupos: (i) Suporte a serviços de TI - determina os meios pelos quais os serviços serão oferecidos e gerenciados, inclui Service Desk, Gestão de Incidentes, Gestão de Problemas, Gestão de Mudanças, Gestão de Configuração e Gestão de Implantação; e (ii) Prestação de serviços de TI - serviços que serão oferecidos, inclui Gerência de Disponibilidade, Gestão de Continuidade de Serviços de TI, Gestão de Capacidade, Gestão Financeira para Serviços de TI e Gestão de Nível de Serviço.

O modelo ITIL, concebido na década de 80 pela Central Computer and Telecommunications Agency (CCTA) tornou-se no início dos anos 90, best practice, tendo sido adotado por diversos países tornando-se referência mundial em 1996. Em 2000, foi criado o Office for Government Commerce (OGC) com intuito de regulamentar o uso do ITIL. Nesse mesmo ano foi definido o Microsoft Operation Framework (MOF) [2]. Em 2001, foram criados o European Examination Institute for Information Science (EXIM) e o Information Systems Examination Board (ISEB), órgãos certificadores dessa metodologia. Par maiores informações vide [5].

\subsection{Modelo COBIT}

No mundo globalizado, a informação e a tecnologia que suportam o negócio e o conhecimento organizacional representam o seu mais valioso recurso. Além disso, num ambiente de negócios altamente competitivo e dinâmico é requerido uma excelente habilidade gerencial, na qual a TI deve suportar as tomadas de decisão de forma rápida, constante e com custos cada vez mais baixos. Nesse contexto, o modelo internacional COBIT pode ser citado como um recurso eficiente para auxiliar o gerenciamento e o controle das iniciativas de TI nas empresas. O modelo COBIT é conhecido como Governança em TI ou IT Governance.

O COBIT é um guia para a gestão de TI recomendado pela Fundação americana Information Systems Audit and Control Foundation (ISACF) [3], que inclui recursos tais como um sumário executivo, um framework, controle de objetivos, mapas de auditoria, um conjunto de ferramentas de implementação e um guia com técnicas de gerenciamento. As práticas de gestão do COBIT são recomendadas pelos peritos em governança de TI que ajudam a otimizar os investimentos de TI e fornecem métricas para avaliação dos resultados. Os modelos de maturidade de governança são usados para o controle dos processos de TI e fornecem um método eficiente para classificar o estágio da organização de TI. A governança de TI e seus processos com o objetivo de adicionar valor ao negócio através do balanceamento do risco e retorno do investimento podem ser classificados numa ordem crescente: (0) Nível inexistente; (1) Inicial/Ad Hoc; (2) Repetitivo, mas intuitivo; (3) Processos definidos; (4) Processos gerenciáveis e medidos e (5) Processos otimizados.

Essa abordagem é derivada de modelos de maturidade para desenvolvimento de software, a saber: Capability Maturity Model Integrated for Software Engineering 
(CMMI-SW), Systems Engineering (CMMI-SE), Integrated Product and Process Development (CMMI-IPPD), todos propostos pelo instituto americano da Carnegie Mellon University - Software Engineering Institute (SEI). Para maiores informações vide [4].

\section{Metodologia Empregada}

Como metodologia empregada neste trabalho foi utilizado o método de modelagem de processos organizacionais partindo-se da premissa que esta é uma abordagem que possibilita o alinhamento entre processos, informações e recursos. Essa abordagem será empregada no tratamento eficaz da informação, provendo tanto facilidades como respaldos formais à tomada de decisão, a qual será norteada pelos objetivos e critérios da instituição envolvida.

A figura 1 representa o esquema da metodologia utilizada onde pode-se verificar que a modelagem de processos foi utilizada como base de ligação entre as melhores práticas do modelo ITIL e os conceitos de governança do modelo COBIT. Essa metodologia objetiva a elevação do nível de maturidade de TI da organização em questão, através da padronização, documentação, divulgação, monitoração e mediação dos processos envolvidos. Atuando dessa forma como uma ponte entre o tratamento da informação e do conhecimento de TI.

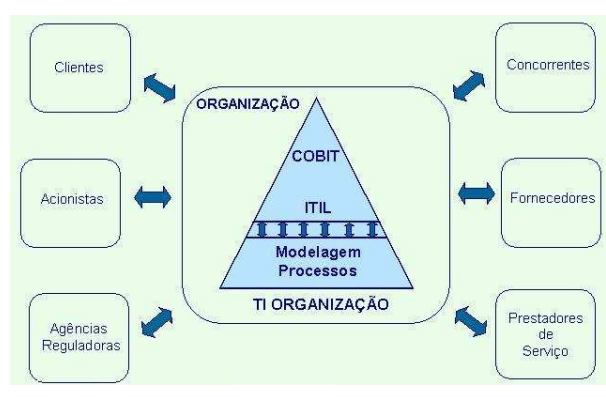

Figura 1. Esquema da Metodologia Utilizada.

A metodologia empregada neste trabalho se utiliza de modelagem de processos como base de melhoria nos padrões de gestão e governança de TI. A modelagem é realizada de forma que cada um dos processos estudados possam ser explodidos em níveis menores, cujo grau de detalhamento contenha informações das tarefas envolvidas em diversos níveis de granularidade. Como exemplo de dados contidos nos repositórios dos modelos de processos podemos citar: recursos humanos e materiais envolvidos, tempos de execução, automaticidade, aplicabilidade, atribuições, sincronismo, condições de parada, entradas e saídas requeridas e a documentação padronizada da execução da tarefa. Essas atividades por sua vez, estão representadas por caminhos de execução que determinam a sua seqüencialidade ou o seu paralelismo. Diversos outros objetos podem ser utilizados, uma vez que representam: tomadas de decisão, dispositivos de entrada e saída e conectores indicativos de loops. Assim que forem levantados todos os elementos envolvidos na modelagem, ou seja, uma vez que esteja populado o repositório de dados do modelo, a ferramenta de modelagem disponibiliza estimativas de custo envolvendo todos os recursos no processo de simulação, os quais podem ser executados em tempo real quando acoplados a uma ferramenta de workflow [1]. 
Neste trabalho, os processos foram inicialmente modelados da maneira mais próxima possível de sua real execução num ambiente de TI através do modelo as-is. De posse do modelo, realizou-se uma análise segundo os conceitos de suporte a serviços de TI do modelo ITIL focando a gestão de incidentes. Dessa forma, pudemos analisar através de diversas simulações o modelo to-be mais adequado.

Dentre os benefícios da Gerência de Incidentes podemos citar: (i) definição formal dos incidentes, tendo por resultado a minimização do impacto no negócio; (ii) melhor utilização dos recursos de suporte; (iii) melhor compreensão do impacto dos incidentes, permitindo priorizações; (iv) informação exata dos incidentes que estão ocorrendo e (v) aumento da disponibilidade de informação para gerência.

\section{Estudo de Caso}

Através da abordagem de modelagem de processos e da metodologia utilizada neste trabalho foi realizado um estudo de caso relacionado à gestão de incidentes em um ambiente real de TI de uma instituição bancária. O ambiente de TI em questão é composto por uma equipe de 15 analistas de informática, divididos em duas sub-equipes de serviços, distribuídas nos turnos noite e madrugada. Essa equipe, juntamente com um grupo de técnicos vinculados a uma empresa prestadora de serviços, é responsável pelos processos de instalação e reinstalação de cerca de 7000 servidores de agências e de postos bancários. Os equipamentos desse ambiente são componentes de uma vasta rede de comunicação de topologia híbrida, a qual possibilita o correto funcionamento dos serviços disponibilizados em mais de 15.000 pontos de atendimentos de clientes espalhados pelo país.

A coleta de informações referentes às tarefas envolvidas, tais como fluxo de atividades, recursos envolvidos, entradas e saídas, procedimentos requeridos, custos, decisões e conhecimentos necessários foi obtida através da experiência pessoal do autor deste artigo no processo de reinstalação de servidores, bem como através do contato com colegas que possuem experiência variando entre 2 meses a 10 anos, além de técnicos da prestadora de serviços e gerentes de setor. Paralelamente, foram realizados levantamentos estatísticos tais como número de interrupções (abortagens), tipos de instalações (1 ou 2 servidores) e motivos de pendências de instalação através dos aplicativos Action Request System (ARS) e Checklist. A coleta foi realizada de maio a junho de 2004.

Os dados obtidos nas simulações desse estudo referem-se a um montante médio diário de 40 atividades de intervenção em servidores, as quais são realizadas fora do expediente bancário. Durante a execução deste trabalho, foi realizado inicialmente um estudo das diversas tarefas envolvidas no processo de instalação e reinstalação de servidores. Tal estudo possibilitou a definição do modelo as-is na ferramenta WBI Workbench, bem como um modelo to-be otimizado utilizando-se dos conceitos de melhores práticas de gestão de TI da biblioteca do ITIL.

\subsection{Modelo as-is}

Esta modelagem foi feita de acordo com o que foi observado no ambiente de trabalho da equipe serviços da noite e da madrugada. Inicialmente, temos uma modelagem em seu mais alto nível de representação apresentado na figura 2. Podemos observar os três principais processos (caixas representadas pela letra $P$ ) que compõe o serviço de reinstalação de servidores dessa organização: (i) procedimento técnico - intervenções locais de instalação 
e configuração de equipamentos; (ii) procedimentos GEPRO (Gerência de Produção) atividades de monitoração/validação das intervenções técnicas realizadas pelo processo anterior e (iii) problema - atividades que tratam de problemas administrativos durante a reinstalação.

$\mathrm{Na}$ figura 2, percebemos que os processos estão ligados por elementos de entrada e saída através de contatos telefônicos entre analistas da GEPRO (0800) e técnicos da empresa prestadora de serviços. Cabe ressaltar também, que os técnicos, caso tenham alguma dificuldade, dispõem além do 0800 (suporte GEPRO), de uma Central de Atendimento local (CAT), para auxiliá-los nas questões técnicas. Desse modo, os processos mostrados na figura 2 revelam que há uma intensa comunicação entre técnicos e analistas do GEPRO via chamada telefônica, ocasionada principalmente por falhas na baixa de matrizes ou por erros durante as tarefas de sincronização de servidores.

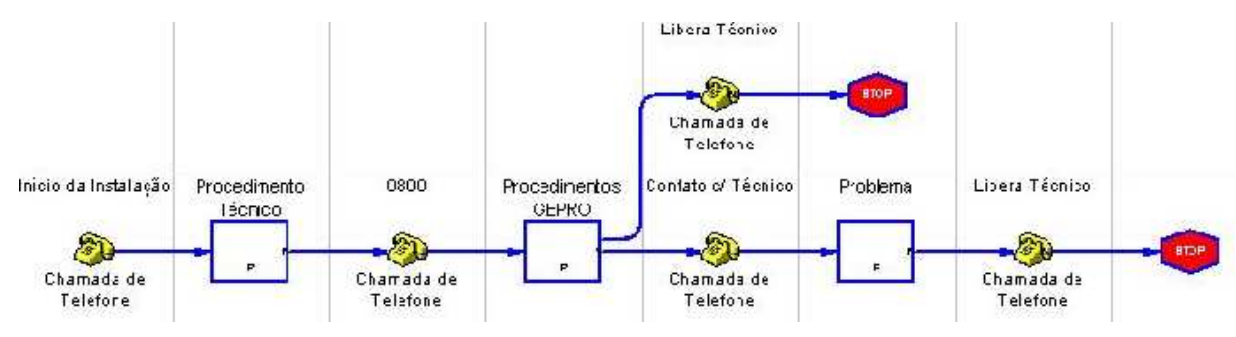

Figura 2. Modelo as-is do procedimentos de reinstalação (high-level).

Nas explosões do modelo mostrado na figura 2 (ilustrações não incluidas no artigo), foram representados: 35 tarefas, 10 requisições de entrada e saída, 15 recursos envolvidos e 22 tomadas de decisão.

\subsection{Modelo to-be}

Nesta modelagem buscou-se otimizar o modelo as-is eliminando-se os gargalos através dos conceitos de gerência de incidentes do modelo ITIL. $\mathrm{O}$ foco foi dado à redução dos custos e tempos de execução das tarefas. Verificou-se que havia muitos gargalos nas tarefas dos analistas GEPRO, implicando em aumento do número de funcionários. Verificouse também, com base nas estatísticas apresentadas pela ferramenta, uma elevada quantidade de tempo desperdiçada no grande número de ligações entre técnicos e analistas. Desse modo, entre as principais alterações sugeridas podemos citar:

- Redução do quadro de funcionários utilizados no processo de reinstalação, para tal sugere-se a utilização de 3 funcionários da equipe de disponibilidade de servidores, os quais facilmente poderiam ser treinados para desempenhar esse serviço;

- Utilização de um Banco de Dados monitorado pela equipe de Internet/Intranet com as versões atualizadas de Migra, Matriz TMF (Terminais de Múltiplas Funções) e Cdac. ${ }^{1}$ Dessa maneira, seriam evitados gastos com o CAT e com ligações desnecessárias. Os arquivos necessários à reinstalação de servidores seriam obtidos diretamente no site;

\footnotetext{
${ }^{1}$ Mídia utilizada para atualizar os servidores com informações referentes a endereços IP's de equipamentos como terminais de atendimento e de auto-atendimento.
} 
- Utilização de apenas um servidor nas agências. Este servidor apresentaria um sistema operacional mais estável como o Linux e a contingência seria diretamente nos servidores de contingência localizados na sede do complexo central de tecnologia.

A figura 3 ilustra as alterações sugeridas acima através da modelagem to-be de mais alto nível. Note que o processo problema da figura 2 foi substituída pelo processo intranet GEPRO, que pode ocorrer em paralelo com o processo reinstalação GEPRO. As explosões do modelo mostrado na figura 3 não estão incluídas no artigo; todavia podemos citar que na representação foram modelados: 13 tarefas, 9 requisições de entrada e saída, 13 recursos envolvidos e 7 tomadas de decisão.

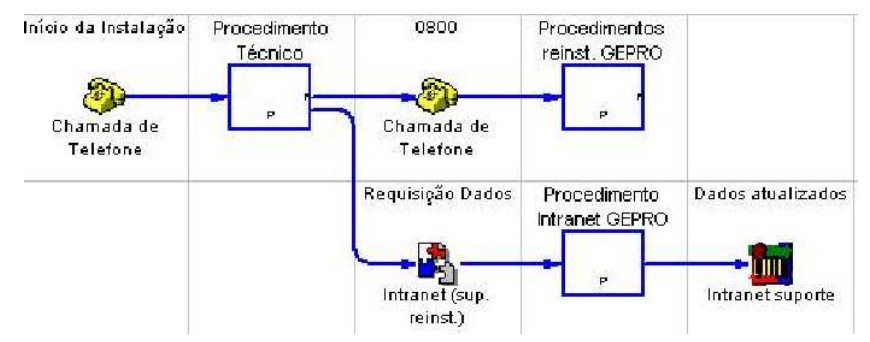

Figura 3. Procedimentos da Reinstalação to-be (High Level).

Como extensão da modelagem do processo de instalação e reinstalação feita, foi elaborada uma outra modelagem que envolve processos que vão desde a compra do equipamento até a sua instalação nas agências da instituição bancária. Esse modelo, abrange outras gerências de TI contemplando atividades que são análogas às gerências do modelo ITIL, e.g. Gerência de Configuração, Problemas e Mudanças.

\section{Conclusões e Trabalhos Futuros}

Este trabalho descreve uma abordagem de modelagem de processos como método de melhoria nos padrões de gestão de TI. Utilizando-se de conceitos e padrões gerencias com funções bem definidas (ITIL) e determinação de métricas para acompanhar o alcance otimizado dos objetivos (COBIT) conseguimos a partir da modelagem as-is a definição do modelo to-be, no qual foi sensível a redução dos custos das atividades e do tempo de execução conforme exibido na tabela 1 .

Tabela 1. Comparação de valores entre modelos as-is e to-be.

\begin{tabular}{|c|c|c|c|c|}
\hline Processo & Valores & Modelo as-is & Modelo to-be & Redução \\
\hline \multirow{2}{*}{ Procedimento Técnico } & Horas & 3.41 & 2.66 & $21,99 \%$ \\
\cline { 2 - 5 } & Custo (\$) & 33.89 & 27.40 & $19,15 \%$ \\
\hline \multirow{2}{*}{ Procedimento Gepro } & Horas & 8.39 & 3.86 & $53,66 \%$ \\
\cline { 2 - 5 } & Custo (\$) & 72.69 & 36.06 & $50,39 \%$ \\
\hline
\end{tabular}

Com relação aos níveis de maturidade do COBIT apresentados na seção 2.2, podemos dizer que através dessa metodologia, partimos de um ambiente no qual foi observado que os processos de instalação/reinstalação de servidores seguiam um padrão regular, mas intuitivo (nível 2), para um estágio no qual os processos passaram a ser documentados e 
comunicados (nível 3), e através da utilização da ferramenta, puderam ser monitorados e medidos (nível 4). O Próximo estágio a ser atingido seria o otimizado, onde teríamos a adoção das melhores práticas de maneira automatizada (nível 5). Conclui-se que o método apresentado ampliou a visibilidade na implantação de conceitos de serviços e seu gerenciamento, permitiu uma maior integração entre as diversas áreas de TI, usuários e prestadores de serviço, permitindo então uma melhor gestão do conhecimento de TI.

Podemos citar que as alterações propostas nesse trabalho já estão sendo adotadas pela instituição: (i) a instalação de somente um servidor Linux nas agências; (ii) a readequação de funcionários no processo de reinstalação de servidores e (iii) a utilização da internet para baixa de mídias pelos técnicos. Como trabalhos futuros, incluímos um estudo mais aprofundado dos modelos ITIL e COBIT, para definição de métodos que possibilitem a gerência de conhecimento empresarial, viabilizando o alinhamento entre os negócios e a área de TI. Julgamos que para tais definições, estudos de caso reais devem ser cada vez mais ampliados.

\section{Referências}

[1] Ferramenta WBI-Workbench http://www14.software.ibm.com/webapp/ download/product. jsp? s=p\&id=RBAR-5 JVJNP. Acesso em 15/01/05.

[2] Documentação MOF/MSF. http: //www.microsoft.com/. Acesso em 20/09/05.

[3] Information Systems Audit and Control Foundation (ISACF). http://www. isaca.org. Acesso em 10/08/04.

[4] Instituto de Governança de TI. http: / /www. itgovernance. org. Acesso em 14/05/04.

[5] Office for Government Commerce (OGC). http: / /www . ogc . gov . uk. Acesso em 14/05/04.

[6] Umit S. Bititci and Daniel Muir. Business process definition: a bottom-up approach. International Journal of Operations \& Production Management, 17(4):365-374, 1997.

[7] Maull R. Bennet J. Weaver A. Smart A. Childe, S. Standard business processes. Working Paper WP/GR/J95010/6, EPSRC Research Report, 1995.

[8] T.H. Davenport. Process Innovation. Harvard Business School Press, Boston, MA., 1993.

[9] Omar A. El Sawy. Redesignig Enterprise Process for e-business. McGraw-Hill, 2002.

[10] Michael Hammer. Reengineering work: Don't automate, obliterate. Harvard Business Review, pages 70-91, July-August 1990.

[11] Michael Hammer and James Champy. Reengineering the corporation: A manifesto for business revolution. Business Horizons, 36(5):90-91, 1993.

[12] David Harvey. Re-engineering: The critical success factors. Technical report, Business Intelligence, January 1995.

[13] Kenneth C. Laudon and Jane P. Laudon. Sistemas de Informação Gerenciais: Administrando a Empresa Digital. Prentice Hall, 2004.

[14] M. Partridge and L. Perren. Achieving competitive advantage. Management Accounting, 71(10):497-508, 1993.

[15] Michael E. Porter. Competitive Advantage : Creating and Sustaining Superior Performance. Free Press Edition, 1985. 